\title{
Guerres et conflits dans les manuels et dans l'enseignement : interprétations, représentations
}

\section{Brigitte Morand}

\section{(2) OpenEdition \\ Journals}

Édition électronique

URL : http://journals.openedition.org/trema/699

DOI : $10.4000 /$ trema.699

ISSN : 2107-0997

Éditeur

Faculté d'Éducation de l'université de Montpellier

Édition imprimée

Date de publication : 1 mars 2008

Pagination : 1-4

ISSN : 1167-315X

\section{Référence électronique}

Brigitte Morand, «Guerres et conflits dans les manuels et dans l'enseignement : interprétations, représentations », Tréma [En ligne], 29 | 2008, mis en ligne le 01 mars 2010, consulté le 22 septembre 2020. URL : http://journals.openedition.org/trema/699 ; DOI : https://doi.org/10.4000/trema.699

Ce document a été généré automatiquement le 22 septembre 2020

Trema 


\title{
Guerres et conflits dans les manuels et dans l'enseignement : interprétations, représentations
}

\author{
Brigitte Morand
}

1 Consacrer un numéro de Tréma au discours sur la guerre dans les manuels scolaires et dans l'enseignement, c'est saisir l'occasion de croiser l'étude d'un sujet d'historiographie très contemporaine, aux multiples enjeux intellectuels et idéologiques, avec un travail de réflexion sur le manuel scolaire en tant qu'objet de recherche.

2 La guerre tient en effet une place importante dans l'histoire des peuples et des nations, elle en constitue souvent un point d'ancrage mémoriel fort, parfois même l'événement (le mythe ?) fondateur. Ce qui ne manque pas de nous renvoyer au débat, bien balisé aujourd'hui, de l'opposition entre histoire et mémoire. Selon Henri ROUSSO, « invoquer la mémoire, c'est invoquer un « je me souviens »; se référer à l'histoire, c'est entonner un «il était une fois». Or, face à l'illusion qui consisterait à confondre ces deux démarches, «l'objet de l'école, et de la plupart des sciences humaines et sociales, est précisément de briser cette illusion $»^{1}$. Il n'en reste pas moins vrai que le "il était une fois " des manuels scolaires n'est pas toujours exempt de cette confusion. Le récit national porté par les manuels de la Troisième République n'avait - il pas pour fonction justement d'inculquer une mémoire nationale, ciment d'une adhésion à la France républicaine ? Mieux encore, ces manuels n'ont - ils pas «fabriqué » la nation? «Si une nation, ce n'est à un moment ou l'autre qu'un livre, alors il est possible que la France ait été la nation du Tour de la France par deux enfants - et de ces lecteurs ", nous dit Patrick $\mathrm{CABANEL}^{2}$. Nous garderons cependant à l'esprit les réserves émises depuis longtemps (RIEMENSCHNEIDER 1984) à propos du rôle des manuels dans la construction de la mémoire collective : la réussite de la «fabrique » d'une nation, ou d'une mémoire, ne saurait probablement se passer d'une adhésion globale de la société à laquelle le projet est destiné ${ }^{3}$. 
3 Aujourd'hui, force est de constater que la concurrence entre histoire et mémoire n'est pas éteinte, même si elle se pose différemment. Ainsi en France le traitement de la Guerre d'Algérie est - il au cœur du problème de la "concurrence des mémoires ». Qu'en est - il dans les programmes et les manuels algériens eux - mêmes? Nous verrons, grâce à Hassan REMAOUN, en quels termes elle se pose, une façon de contribuer à l'historiographie de la guerre "d'une rive à l'autre", selon le mot de Benjamin STORA ${ }^{4}$.

4 Car la guerre est aussi un objet d'histoire renouvelé, comme le montrent l'historiographie récente de la Première Guerre Mondiale et la controverse dont elle fait l'objet ${ }^{5}$. Le discours sur la guerre est donc un excellent modèle pour étudier la façon dont les manuels répercutent l'évolution des savoirs universitaires et comment ils rendent compte, ou non, des débats qui l'agitent ${ }^{6}$. Si en France le concept de " culture de guerre » est au centre des controverses sur la Grande Guerre, Rainer BENDIK nous montre que, côté allemand, le traitement des responsabilités du conflit dans les manuels reflète également, bien qu'avec retard, l'évolution historiographique. Plus que la Seconde Guerre Mondiale elle - même, c'est au travers de la Première, et autour de « l'image de l'ennemi » que se joue la question de l'identité collective et la justification des régimes en place, de part et d'autre du rideau de fer. De même, Bertrand LÉCUREUR explore la façon dont s'opère, à propos du second conflit mondial cette fois, la transition didactique des aspects les plus récents de l'historiographie allemande. Et c'est une "autre Allemagne ", celle des résistants au nazisme, qui est mise au jour. À l'heure où les derniers poilus disparaissent, et où le manuel franco - allemand est devenu réalité, ces deux exemples montrent à quel point l'histoire, dans les manuels scolaires aussi, se raconte au présent.

5 La guerre renvoie également à un imaginaire: image de l'autre, image de soi, elle construit et véhicule des représentations. C'est l'occasion de rappeler que «Un manuel, ce n'est pas seulement que du texte, organisé en chapitres selon une progression d'apprentissage. Un manuel comporte une part, plus ou moins grande, d'iconographie: schémas, tableaux, dessins, reproductions d'oeuvres d'art, photos... » ${ }^{7}$ Ainsi du conflit Est - Ouest (la «Guerre Froide »), tel qu'il apparait dans les manuels français de Terminale : à côté du récit linéaire du déroulement des événements, les images fournies par les manuels fonctionnent en réseau, se répondent l'une l'autre, et leur lecture s'effectue de façon simultanée. Ces images, toujours les mêmes, apparaissent aussi, au cours du temps, avec une fréquence remarquable dans les manuels. Appuyées par le texte - auteur, ou venant illustrer celui - ci, elles représentent de véritables stéréotypes (l'avion du blocus de Berlin), ou contribuent à la diffusion ambiguë de mythes (la photographie de Yalta).

Interprétations, représentations: nous voyons comment étudier la façon dont les manuels racontent la guerre oblige à tenir compte de la complexité du manuel lui même en tant qu'objet culturel.

7 Résultant des différentes logiques et processus qui concourent à son élaboration ${ }^{8}$, il obéit d'abord à une commande institutionnelle, et se fait ainsi, comme le remarque Alain CHOPPIN (1992), «le principal vecteur des valeurs que transmet l'institution scolaire ", dont les choix "obéissent à des objectifs politiques, moraux, religieux, esthétiques, idéologiques, le plus souvent implicites. ${ }^{9}$

8 Objet commercial (du moins dans les pays où il existe réellement un marché dans ce domaine), son contenu est conditionné par des considérations économiques (attentes 
supposées ou réelles des enseignants prescripteurs, coûts de production...) qui peuvent également en infléchir le discours.

Support de transposition didactique, il convient de s'interroger sur la façon dont le manuel rend compte de l'évolution des savoirs universitaires, et des interprétations idéologiques et épistémologiques qui nourrissent sa construction, ainsi que sur la façon dont il répercute les représentations qui circulent au sein de la société dans laquelle il est produit et utilisé.

D'une histoire nationaliste à une histoire critique, du récit patriotique vers la construction du citoyen éclairé, c'est le parcours auquel nous convient Hassan REMAOUN, Rainer BENDIK, et Bertrand LÉCUREUR.

11 Mais si la guerre est un événement fondateur, elle peut aussi, comme je me propose de le montrer à propos de la Guerre Froide, bouleverser l'image traditionnelle de soi, obligeant du même coup à une douloureuse recomposition de l'image du monde.

Enfin, il est temps de lever une ambiguïté : si jusque - là nous avons parlé du discours sur la guerre dans les manuels, avouons qu'en réalité les manuels généralement ne racontent pas la guerre, ils racontent des guerres. "La connaissance des conflits a longtemps constitué le socle même de l'enseignement de l'histoire ", nous dit Evelyne FARCY MAGDENEL, mais nous sommes loin d'un enseignement qui porterait sur le phénomène de la guerre en lui - même. Or les programmes préconisent aujourd'hui une Éducation à la Défense : cela pourrait - il, dans le cadre d'une nouvelle éducation à la citoyenneté, être l'occasion d'amener les élèves à une plus grande intelligibilité du monde, dans le cadre d'un enseignement qui conduirait aussi à « faire progresser la paix »?

En effet, que se passe $\mathrm{t}$ - il quand, comme c'est le cas aujourd'hui, en France comme ailleurs, «l'école n'en parle pas»? Quelles images les élèves peuvent - ils alors construire des guerres du présent, et par conséquent du monde dans lequel ils vivent ? N'est - il pas préférable, comme nous le propose Jean - Pierre BRANCHEREAU en conclusion d'une enquête qu'il a menée auprès d'élèves de classes primaires, que l'école propose aux élèves une réflexion sur ce thème, plutôt que de les laisser démunis face à l'abondance des images et sans outils pour les décoder?

\section{NOTES}

1. ROUSSO, H. (2002). "La mémoire et l'histoire ", Apprendre et enseigner la guerre d'Algérie et le Maghreb contemporain, pp.152-153. Les Actes de la DESCO, CRDP de Versailles.

2. CABANEL, P. (2007). « La nation est un livre. Les tours de la nation par les manuels dans l'Europe du XIXe siècle ", VERDELHAN-BOURGADE, M, BAKHOUCHE, B, BOUTAN P \& ETIENNE, R. Les manuels scolaires, miroirs de la nation, p. 22. Paris : L'Harmattan.

3. RIEMENSCHNEIDER, R. (1984). « La confrontation internationale des manuels. Contribution au problème des rapports entre manuels d'histoire et mémoire collective ", H. MONNIOT (dir). Enseigner l'histoire. Des manuels à la mémoire. Travaux du colloque Manuels d'histoire et mémoire collective. UER de didactique des disciplines, Univ Paris 7, Peter LANG. Rainer RIEMENSCHNEIDER émet «l'hypothèse que les manuels scolaires ont un effet certain lorsqu'ils sont en accord avec un vécu réel 
auquel la jeunesse scolaire peut se référer pour donner vie à l'abstraction des textes lorsque le vécu du quotidien et le message du manuel baignent dans le même univers de normes affectives et cognitives. Dans ce cas les manuels peuvent avoir un effet de renforcement, mais qui est toujours secondaire à une prédisposition créée en dehors de l'école et de son enseignement. Ce sont ces rapports du vécu quotidien avec le contenu de l'enseignement qu'il faut à mon avis cerner avec un maximum de précision. »

4. STORA, B. "Repère sur l'historiographie algérienne de la guerre ", Apprendre et enseigner la guerre d'Algérie et le Maghreb contemporain, p. 35. Les Actes de la DESCO, CRDP de Versailles.

5. Faut-il, pour comprendre comment des millions d'hommes ont «tenu " dans l'horreur des combats, avancer le concept de «culture de guerre », comme le font les historiens de l'Historial de PÉRONNE Jean-Jacques BECKER, Annette BECKER et Stéphane AUDOIN ROUZEAU ? Ce n'est pas l'avis de Nicolas OFFENSTADT ou Frédéric ROUSSEAU pour qui « si l'on peut aisément constater que s'impose dès 1914 un discours de guerre, public, auquel peu de contemporains échappent, la question des croyances et des pratiques est assurément plus complexe. " (OFFENSTADT, N. \& ROUSSEAU, F. et al. (2004). " À propos d'une notion récente, la « culture de guerre », Guerres, paix et sociétés, 1911-1946, Neuilly : Atlande.

6. Parlant de Première Guerre Mondiale dans les manuels français, N. OFFENSTADT estime par exemple que "Quand il y a un discours historiographique marqué, c'est surtout celui-là qu'on trouve au niveau du lycée; pour les manuels du collège, la dimension historiographique forte est absente. C'est une question qui se joue donc au lycée et pas du tout dans la tension ni dans la dialectique mais dans l'importation directe des concepts de l'Historial de Péronne dans l'écriture de l'histoire, parfois même de façon caricaturale. " Benoît FALAIZE \& Olivier ABSALON la Grande Guerre dans les manuels scolaires. Entretien avec Nicolas OFFENSTADT, site INRP : http://ecegh.inrp.fr

7. VERDELHAN-BOURGADE, M. Les manuels scolaires, miroirs de la nation? Avant propos p. 9. Paris : L'Harmattan.

8. Comme le dit Nicole LUCAS, «le manuel scolaire est multipolaire : il est le point de convergence de la recherche, de la communication, de la découverte, de la pédagogie, de l'institution et des spécialistes. " LUCAS, N. (2001). Enseigner l'histoire dans le secondaire. Manuels scolaires et enseignement depuis 1902, p. 235. Presses universitaires de Rennes.

9. CHOPPIN, Al. (1992). Les manuels scolaires : histoire et actualité. Paris : Hachette Education.

\section{AUTEUR}

\section{BRIGITTE MORAND}

Docteur en histoire contemporaine. CEDRHE, IUFM de Montpellier brigitte.morand@free.fr 